\title{
Mit Krücken hinters Steuer?
}

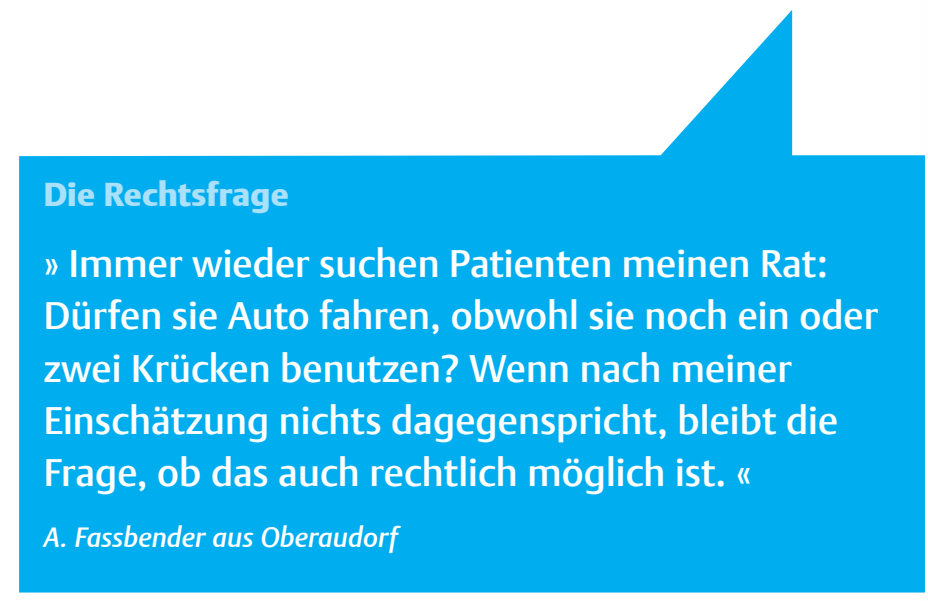

Die Antwort unseres Experten

Wie so oft in der Juristerei lautet die Antwort auf diese Frage: „Es kommt darauf an. " Gemäß § 2 der Fahrerlaubnis-Verordnung darf am Verkehr nur teilnehmen, wer sich trotz körperlicher oder geistiger Beeinträchtigungen sicher im Verkehr bewegen kann. Diese Vorschrift gilt für Kraftfahrer, aber auch für Fußgänger und Fahrradfahrer. Muss oder möchte ein körperlich oder geistig beeinträchtigter Patient dennoch Auto fahren, muss er oder ein für ihn Verantwortlicher dafür sorgen, dass er andere nicht gefährdet. Das gelingt zum Beispiel mit geeigneten Hilfsmitteln an Fahrzeugen, Prothesen, einer Begleitperson oder dem Tragen von Abzeichen oder Kennzeichen. Das heißt, Bewegungseinschränkungen beeinträchtigen nicht zwangsläufig die Fahrsicherheit. Im Einzelfall können sie aber dazu führen, dass ein Patient bestimmte Fahrzeuge nicht bzw. nur mit besonderen Umbauten führen kann, die seine Einschränkung kompensieren. Besitzt der Patient in diesem konkreten Fall ein Automatik-Auto und kann das rechte
Bein uneingeschränkt einsetzen, spricht nichts dagegen, dass er damit Auto fährt.

Grundsätzlich gehört es nicht zu den Aufgaben eines Physiotherapeuten, die Fahrtauglichkeit eines Patienten zu überprüfen. Fällt jedoch ins Auge, dass dieser eingeschränkt fahrtauglich ist, muss ihn der Therapeut darauf hinweisen. Ist der Patient nicht einsichtsfähig, muss der Therapeut die Teilnahme am Straßenverkehr verhindern. Zur Sicherheit sollte man in derartigen Fällen oder bei berechtigten Zweifeln einen Kollegen hinzuziehen und den Hinweis dokumentieren. Vorrangig ist jedoch der behandelnde Arzt dazu verpflichtet, den Patienten auf die eingeschränkte Fahrtauglichkeit hinzuweisen. Kommt es aufgrund von Krankheit oder Medikamenten zu einem Unfall, kann auch der Arzt haften, der seinen Hinweispflichten nicht nachgekommen ist. Karsten Bossow

\section{$\Rightarrow$ Wirft auch Ihr Berufsalltag rechtliche Fragen auf? Dann schreiben Sie an Simone.Gritsch@thieme.de.}

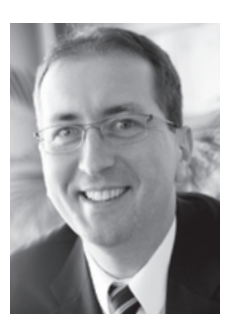

Karsten Bossow ist seit 1999 Rechtsanwalt mit den Tätigkeitsschwerpunkten Arbeits-, Medizin- und Sozialrecht. Seit 2003 ist er Fachanwalt für Arbeitsrecht und seit 2010 Fachanwalt für Medizinrecht. 